\title{
Synthesis, Characterization, and Biodegradation Studies of Poly(1,4-cyclohexanedimethylene-adipate-carbonate)s
}

\author{
Ajay S. Chandure, ${ }^{1}$ Ganesh S. Bhusari, ${ }^{2}$ and Suresh S. Umare ${ }^{2}$ \\ ${ }^{1}$ Metlok Pvt. Ltd, Kalmeshwar, Nagpur 441 501, India \\ ${ }^{2}$ Department of Chemistry, Visvesvaraya National Institute of Technology (VNIT), Nagpur 440 010, India \\ Correspondence should be addressed to Ganesh S. Bhusari; ganesh24bhusari@gmail.com
}

Received 14 January 2014; Accepted 16 February 2014; Published 25 March 2014

Academic Editor: Yves Grohens

Copyright (c) 2014 Ajay S. Chandure et al. This is an open access article distributed under the Creative Commons Attribution License, which permits unrestricted use, distribution, and reproduction in any medium, provided the original work is properly cited.

\begin{abstract}
Aliphatic/alicyclic poly(1,4-cyclohexanedimethylene-adipate-carbonate)s (PCACs) were synthesized by a transesterification from 1,4-cyclohexamethylendimethanol (1,4-CHDM), adipic acid (AA), diethyl carbonate (DEC), and titanium butoxide Ti(OBu) as a transesterification catalyst. The synthesized PCACs were characterized by the Fourier transform infrared (FTIR), X-ray diffraction analysis (XRD), solubility, solution viscosity, gel permeation chromatography (GPC), differential scanning calorimetry (DSC), thermogravimetric analysis (TGA), and scanning electron microscope (SEM) for their structural, physical, thermal, and morphological investigation. The structure of synthesized PCACs was confirmed by FTIR. All TGA curves of PCACs shows $10 \%$ weight loss above $270^{\circ} \mathrm{C}$, and they reveal good thermal stability. Biodegradability of PCACs was investigated by hydrolytic degradation at (pH 7.2 and 11.5), enzymatic degradation using Rhizopus delemar lips at $37^{\circ} \mathrm{C}$ in phosphate buffer solution (PBS), and soil burial degradation at $30^{\circ} \mathrm{C}$. The hydrolytic degradation shows the greater rate of weight loss in PBS at pH-11.5 than pH-7.2. The hydrolytic and soil burial degradation shows faster rate of weight loss as compared to enzymatic degradation. Biodegradation rate of PCACs follows the order: PCAC-20 > PCAC-40 > PCAC-60. SEM images show that degradation occurred all over the film surface, creating holes and cracks. These biodegradable PCACs may be able to replace conventional polymer in the fabrication of packaging film in near future.
\end{abstract}

\section{Introduction}

In recent times, synthetic plastics have attracted much more attention due to their versatility and applicability in various fields. Out of total plastic production, $41 \%$ of synthetic plastics are used in packing industries, and $47 \%$ of them are, in particular, used for packing of foodstuffs [1]. These plastics are generally made from nonrenewable petroleum-based resources, which are nonbiodegradable in nature and create global environmental problems [2]. This limitation can be overcome by the development of ecofriendly biodegradable polymers. Also, the biodegradable polymers have attracted more attention as green materials due to their wide applicability in drug delivery systems, artificial implants tissue engineering, and food packaging [3]. Among the synthetic polymers, poly(ester-carbonate)s (PECs) provide the alternative as they combine the features of biodegradability, biocompatibility, and physicochemical properties $[4,5]$ comparable with many conventional and nonbiodegradable polymers such as polyethylene (PE) and polypropylene (PP).

Recently, PECs derived from ring-opening polymerization of cyclic monomers have been extensively studied for environmentally benign materials and biomedical applications $[6,7]$. Various researchers have reported [79] the synthesis of aliphatic PECs by dichloroformate synthesis and ring-opening polymerization of cyclic carbonates such as ethylene carbonate, trimethylene carbonate, and 2,2-dimethyltrimethylene carbonates. But, PECs via the dichloroformate synthesis required highly expensive and environmentally toxic/hazardous phosgene as chainextension/coupling agents $[8,9]$. The ring-opening polymerization of a lactone or cyclic carbonate needs extremely pure monomers and anhydrous conditions as well as long 


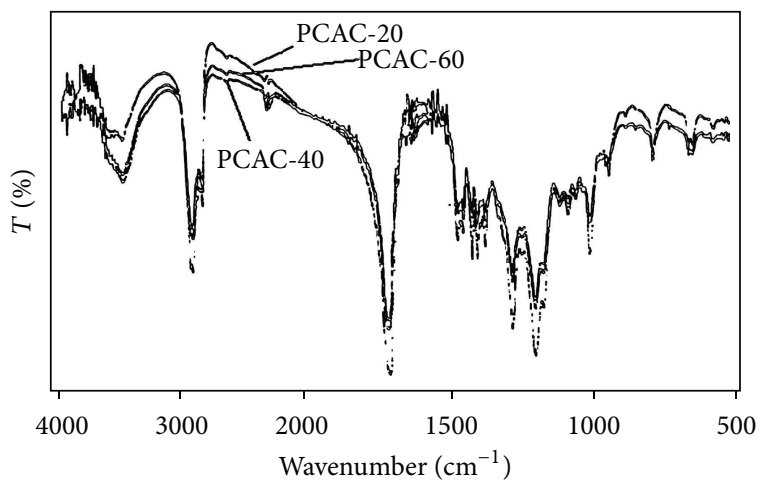

FIgURE 1: FTIR spectra of PCACs.

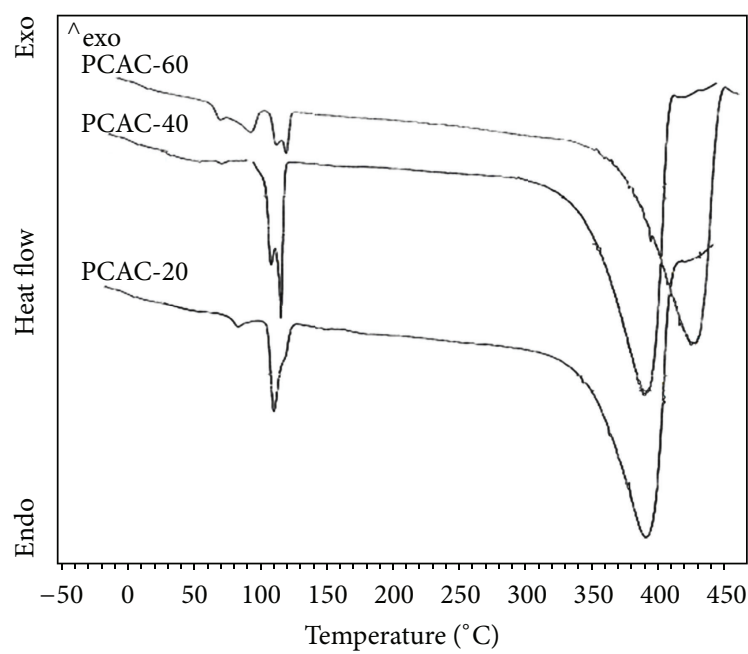

FIgURE 2: DSC thermogram of PCACs.

reaction time. Furthermore. the polymerization catalyst may be contained in the resultant polymer and needs an additional purification procedure for medical applications [10]. The transesterification of a diol with a monomeric carbonate may be one of the feasible processes to obtain biodegradable PECs [11].

In the present paper, we describe the synthesis of biodegradable aliphatic/alicyclic PCACs from 1,4-CHDM, AA (which can be obtained from renewable resources), and DEC in the presence of a transestrification catalyst $\mathrm{Ti}(\mathrm{OBu})_{4}$ at $210^{\circ} \mathrm{C}$. The synthesized PCACs were characterized by FTIR, XRD, solubility, solution viscosity, GPC, DSC, TG, and SEM. The hydrolytic degradation in phosphate buffer $(\mathrm{pH}=7.2$ and 11.2), enzymatic degradation using Rhizopus delemar lipase in phosphate buffer $(\mathrm{pH}=7.2)$, and soil burial degradation studies were used to investigate the biodegradation of PCACs.

\section{Experiment}

2.1. Materials. 1,4-Cyclohexamethylendimethanol (1,4CHDM; Fluka, cis-trans 98\%), adipic acid (AA; 99\%), diethyl carbonate (DEC; 99.5\%), titanium butoxide $\mathrm{Ti}$ $(\mathrm{OBu})_{4}(97 \%), \mathrm{Na}_{2} \mathrm{HPO}_{4}$ (99.9\%), phosphate buffer solution

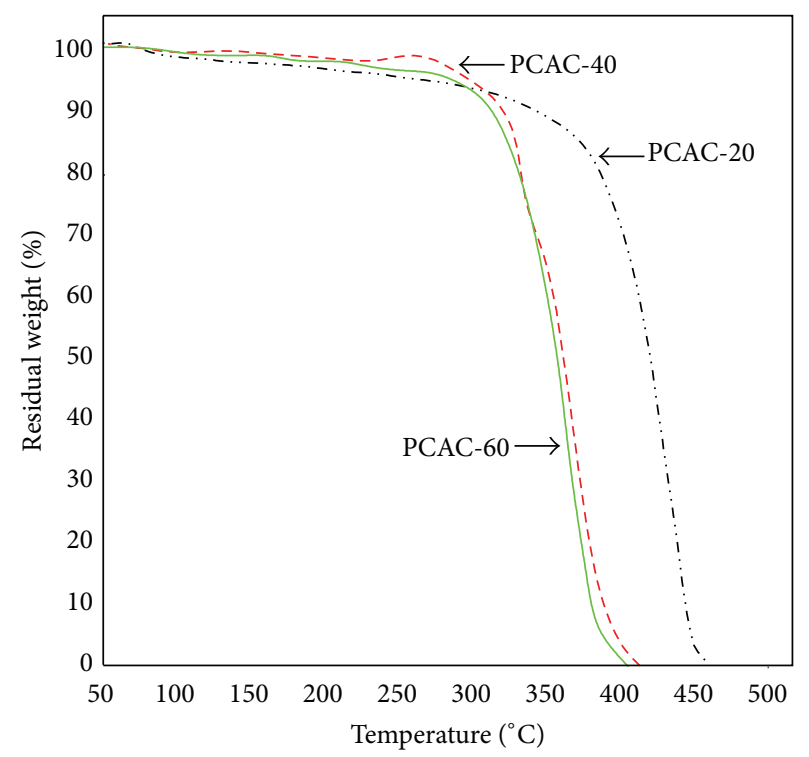

FIgUre 3: TG curve of PCACs.

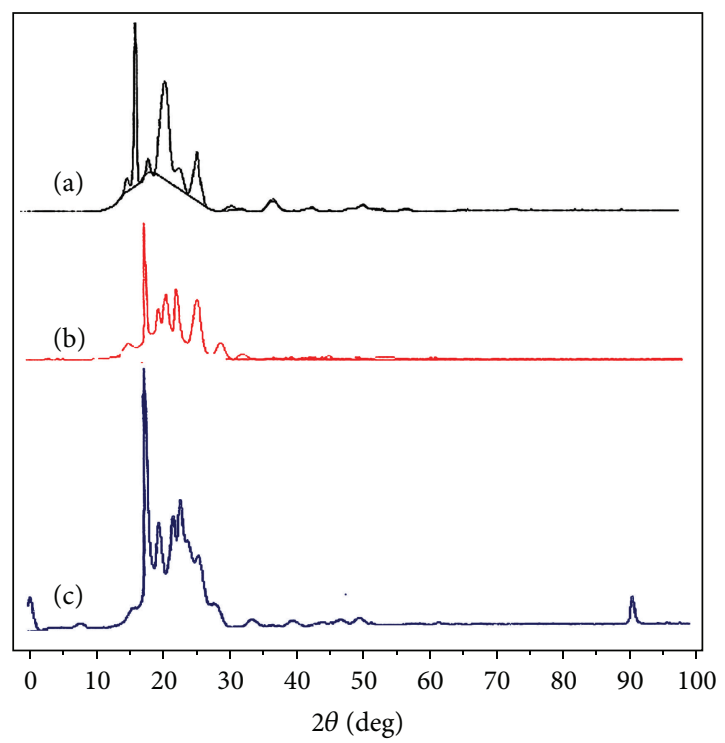

FIgure 4: XRD pattern of (a) PCAC-20, (b) PCAC-40, and (c) PCAC-60.

(PBS; pH 7.2 \& 11.2), and enzymes Rhizopus delemar lipase (Fluka, $0.73 \mathrm{U} / \mathrm{mg}$ ) were purchased from the Sigma-Aldrich Chemical Co., Germany. Methanol, chloroform, acetone, 1-methyl-2-pyrrolidone (NMP), tetrahydrofurane (THF), $\mathrm{N}$, N-dimethylformamide (DMF), dimethylsulphoxide (DMSO), $\mathrm{NaOH}$, and so forth, were of E-Merck, (India) spectroscopic grade. All the reagents were used as received without further purification.

2.2. Synthesis of PCACs. PCACs were synthesized in the bulk by transesterification reaction [12-14] in the presence of $\mathrm{Ti}(\mathrm{OBu})_{4}$ as transesterification catalyst. The polycondensation reactor was a three-necked flask equipped with 


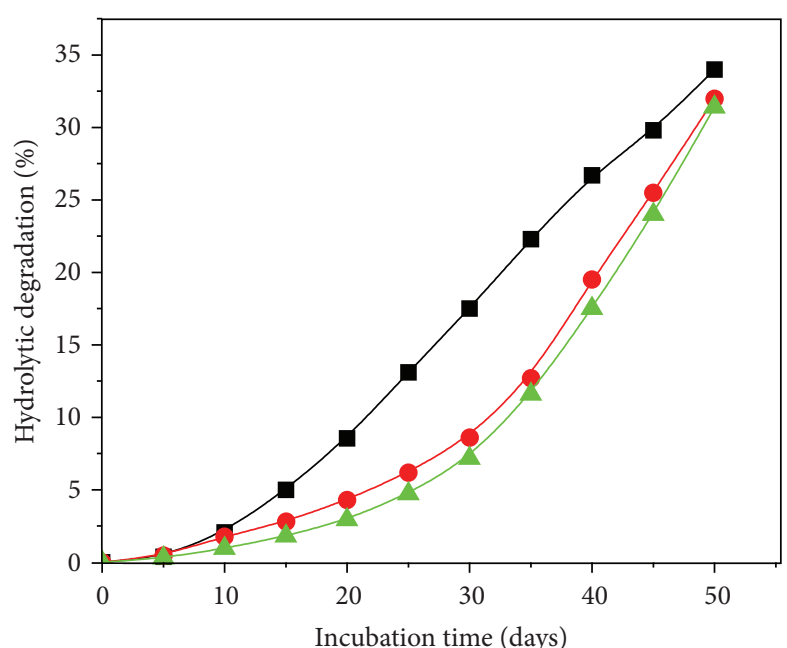

(a)

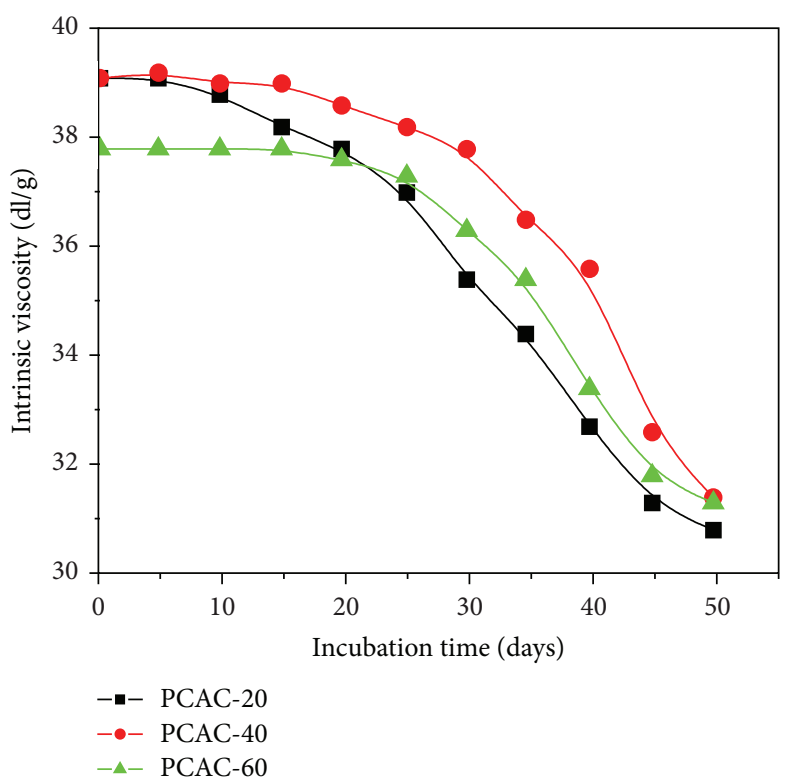

(c)

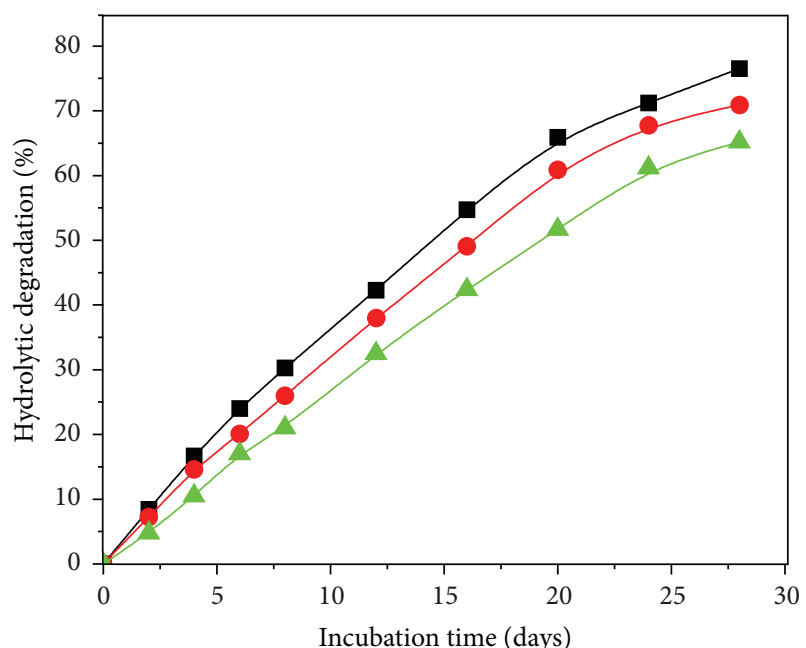

(b)

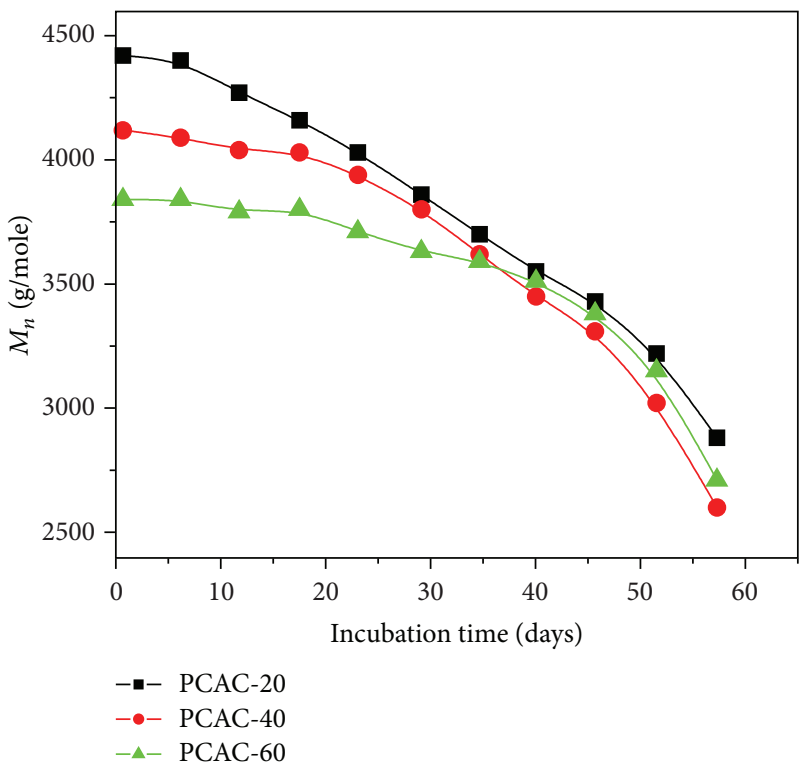

(d)

Figure 5: (a) Weight loss at pH 7.2 during hydrolytic degradation of PCACs with incubation time. (b) Weight loss at pH 11.5 during hydrolytic degradation of PCACs with incubation time. (c) Variation in intrinsic viscosity at $\mathrm{pH} 7.2$ during hydrolytic degradation of PCACs with incubation time. (d) Variation in number average molecular weight at $\mathrm{pH} 7.2$ during hydrolytic degradation of PCACs with incubation time.

a magnetic stirrer, a condenser and thermometer, nitrogen gas inlet, and outlet for continuous flow of nitrogen. The PCACs were synthesized by charging AA (116.91 g; 0.8 mole), DEC (23.62 g; 0.2 mole), 1,4-CHDM (158.64 g; 1.1 mole), and $\mathrm{Ti}(\mathrm{OBu})_{4}(0.3 \mathrm{~g} ; 0.882 \mathrm{mmole})$ into the reactor. The reaction mixture was purged with nitrogen and it was heated at $160^{\circ} \mathrm{C}$ for $4 \mathrm{~h}$. The water formed during the reaction was distilled off. In the second phase of the reaction, the reaction mixture was subsequently heated to final temperature of $210^{\circ} \mathrm{C}$ under reduced pressure $(<0.5$ torr) for $1 \mathrm{~h}$ in order to distill off the trace of water. The obtained PCAC was dissolved in a minimum amount of chloroform and reprecipitated with a tenfold amount of ice-cold methanol with vigorous stirring. The reaction mixture was filtered and washed with methanol, and the precipitate was then dried at $60^{\circ} \mathrm{C}$ under reduced pressure. The product obtained was a brownish solid powder and the yield was $85 \%$. The PCAC- 20 , PCAC- 40 , and PCAC60 were synthesized by varying the mole percentage of AA and DEC at constant the 1,4-CHDM mole ratio using a similar procedure.

\section{Characterization}

The solubility of the PCACs was tested by dissolving $0.25 \mathrm{~g}$ of polymer in $50 \mathrm{~mL}$ solvent such as water, chloroform, THF, DMSO, NMP, DMF, acetone, $n$-hexane and ethyl ether, ethyl acetate, and ethanol. The mixture was stirred for $30 \mathrm{~min}$ and kept for $4 \mathrm{~h}$ at room temperature $\left(30^{\circ} \mathrm{C}\right)$ and the solubility 


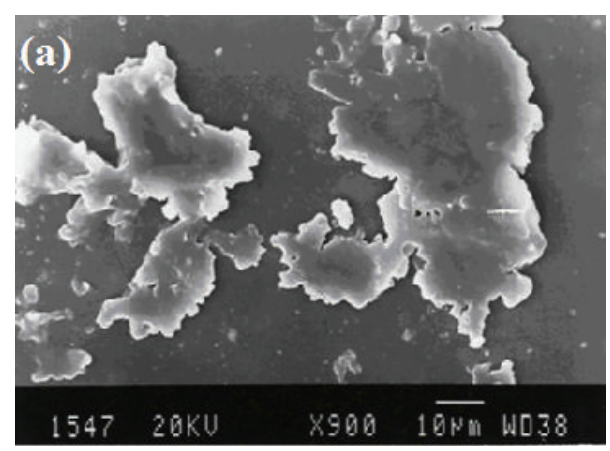

(a)

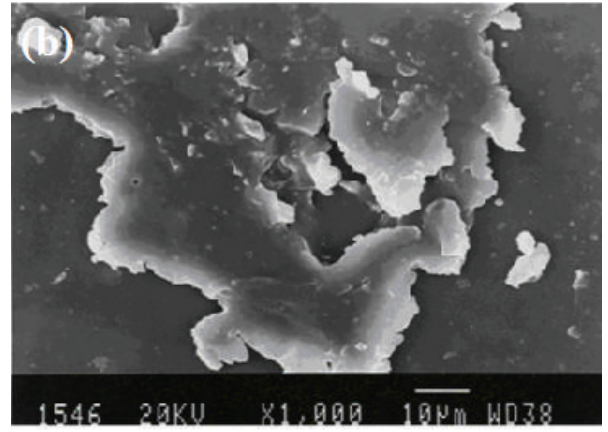

(b)

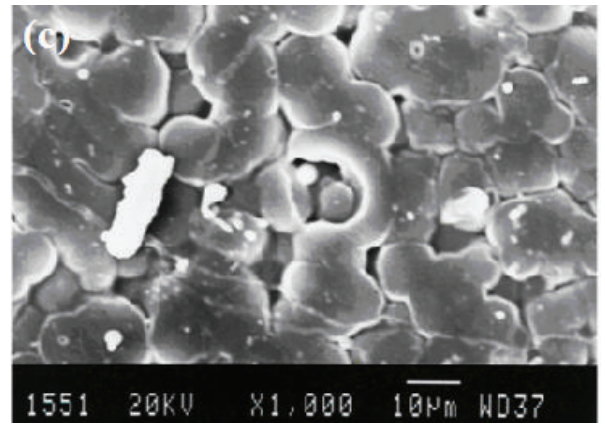

(c)

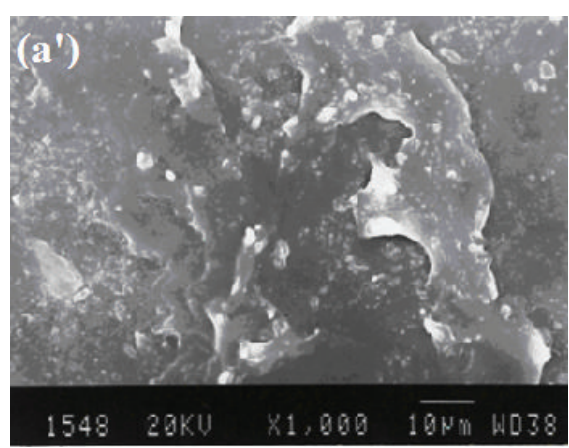

$\left(a^{\prime}\right)$

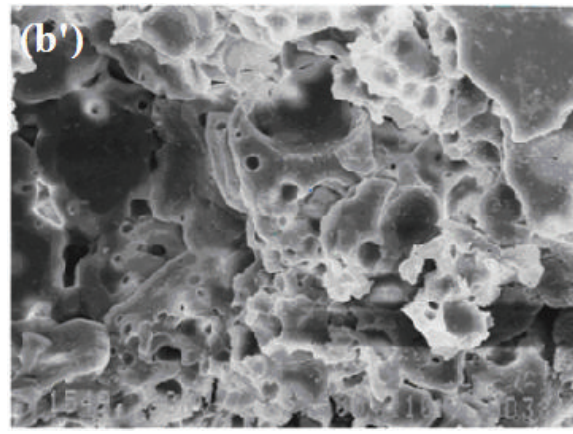

$\left(b^{\prime}\right)$

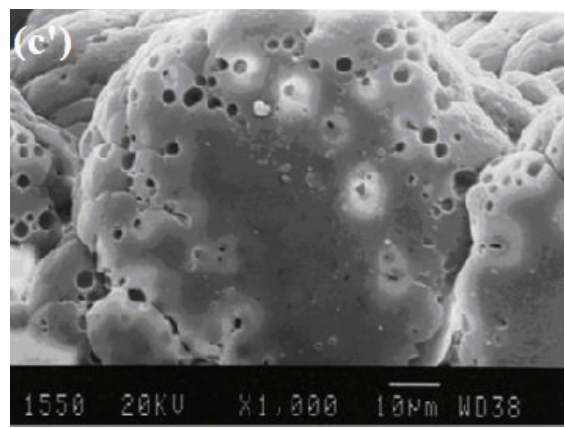

$\left(c^{\prime}\right)$

FIGURE 6: SEM micrographs of hydrolytic degraded PCACs films at pH 7.2. Original films: (a) PCAC-20, (b) PCAC-40 and (c) PCAC-60. After 40 day of degradation: $\left(a^{\prime}\right)$ PCAC-20, $\left(b^{\prime}\right)$ PCAC-40 and $\left(c^{\prime}\right)$ PCAC-60.

observations were recorded as average of three independent readings. The FTIR spectra of PCACs was performed by $\mathrm{KBr}$ pellet technique on Shimadzu FTIR- 8101A, Japan. The solution viscosity $\left[\eta_{\text {int }}\right]$ of the original and degraded PCACs film samples in chloroform was measured at $30^{\circ} \mathrm{C}$ using a Tuan-fouss viscometer. From the time flow of solvent and the solution, the intrinsic viscosity $\left[\eta_{\text {int }}\right]$ was calculated as the average of three independent readings. The molecular weights and their distribution were determined by gel permeation chromatography (GPC). The instrument was equipped with a refractive index detector (RI) and PLGel $5 \mu \mathrm{m}$ mixed$\mathrm{D}$ type column. THF was used as mobile phase at a flow rate of $1.0 \mathrm{~mL} / \mathrm{min}$ and polystyrene solution in THF as the calibration standard. $20 \mu \mathrm{L}$ of a $1 \%$ polymer solution in THF was injected in all the cases. The number average $\left(M_{n}\right)$ and weight average molecular weights $\left(M_{w}\right)$ were calculated from the calibration curve.

Differential scanning calorimetry (DSC) and thermogravimetry (TG) analysis of original and the degraded PCACs sample were conducted on a Mettler Toledo-822 analyzer and Perkin-Elmer, Diamond instrument, at a heating rate of $10^{\circ} \mathrm{C} / \mathrm{min}$ under nitrogen atmosphere in the temperature range -50 to $450^{\circ} \mathrm{C}$ and 28 to $600^{\circ} \mathrm{C}$ for DSC and TGA, respectively. XRD analysis was performed on original and degraded PCACs film samples using an " $\mathrm{X}$ " Pert PRO PANalytical diffractometer with a $\mathrm{CuK} \alpha$ target tube. The measurements of the samples were performed over the range from $5^{\circ}$ to $80^{\circ}$. From the XRD data, the degree of crystallinity was calculated as the ratio of the total intensities of the crystalline reflections and the overall diffraction pattern area. 


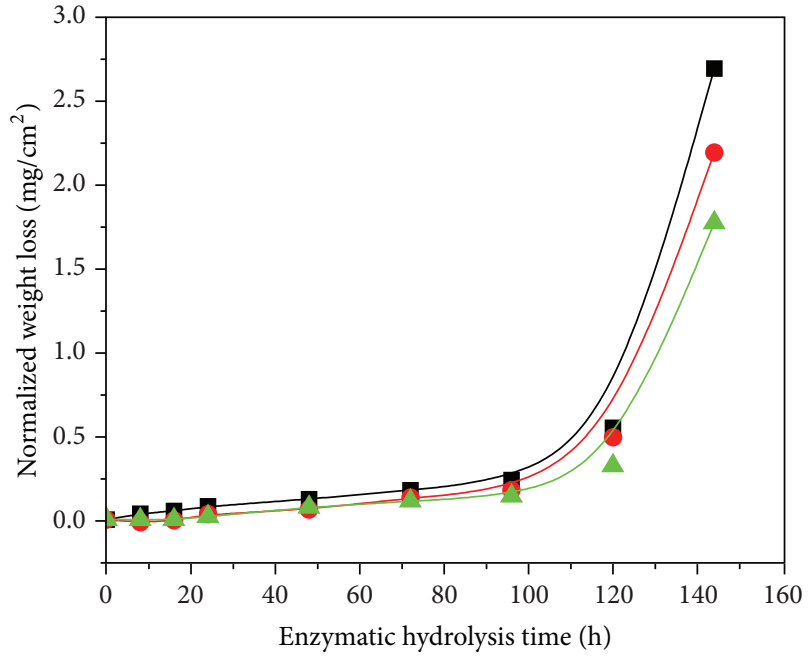

(a)

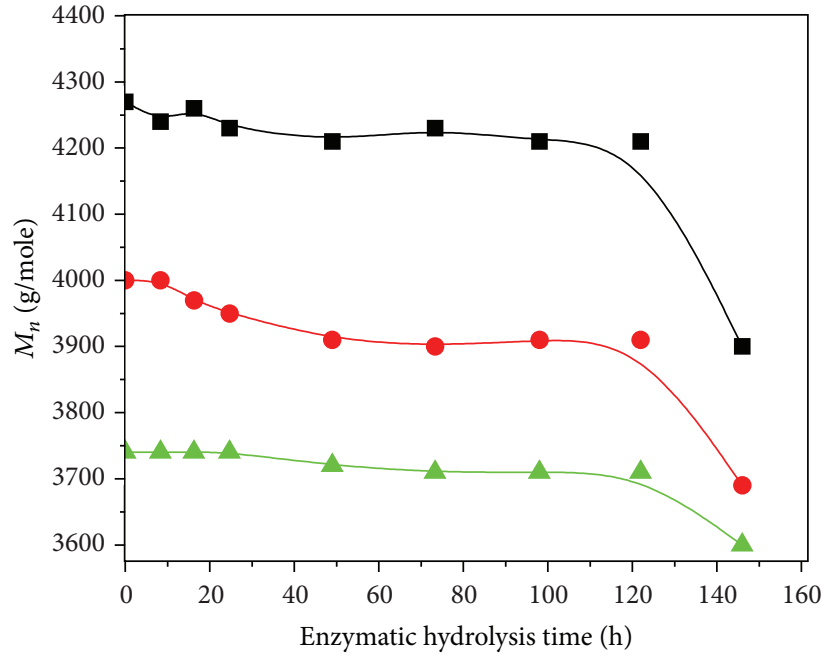

(b)

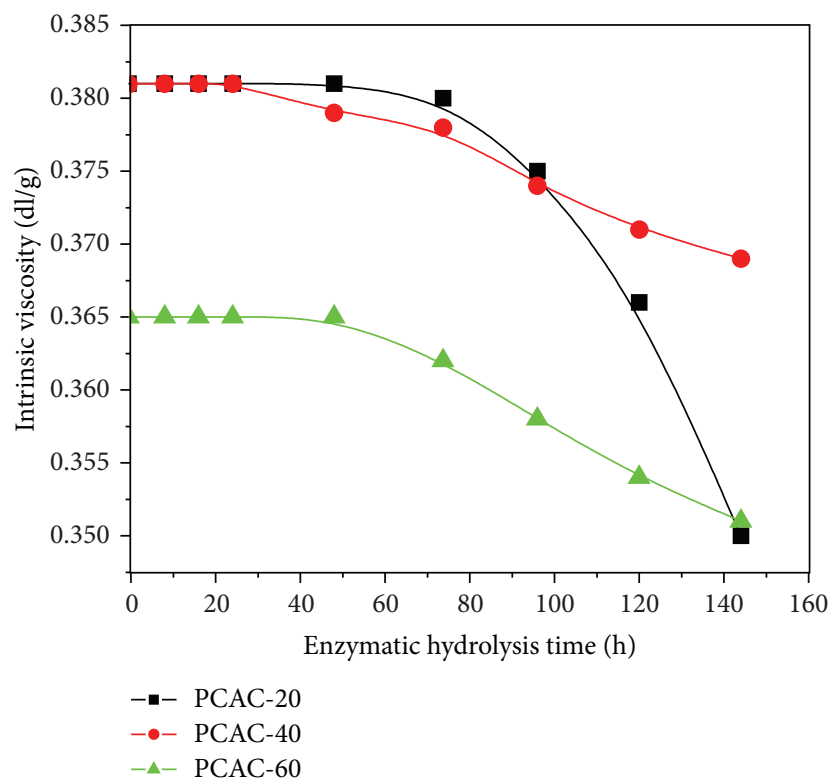

(c)

FIGURE 7: (a) Normalized weight loss per unit area with incubation time during enzymatic degradation of PCACs. (b) Variation in $M_{n}$ with incubation time during enzymatic degradation of PCACs. (c) Variation in $\left[\eta_{\text {int }}\right]$ with incubation time during enzymatic degradation of PCACs.

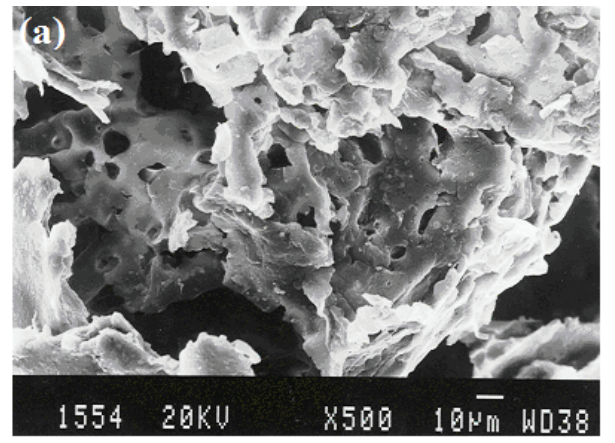

(a)

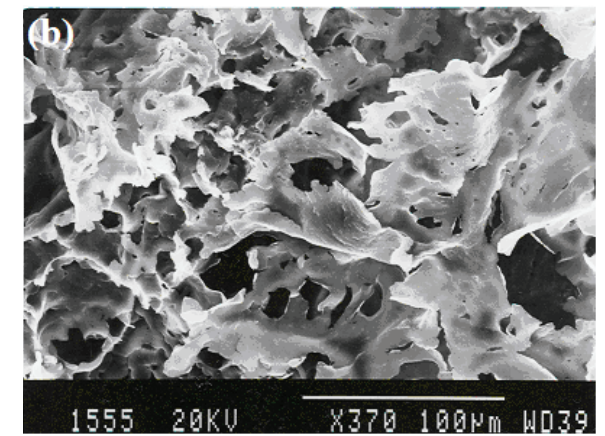

(b)

FiguRE 8: SEM micrographs of PCAC-60 after (a) $96 \mathrm{~h}$ and (b) $144 \mathrm{~h}$ of enzymatic degradation. 


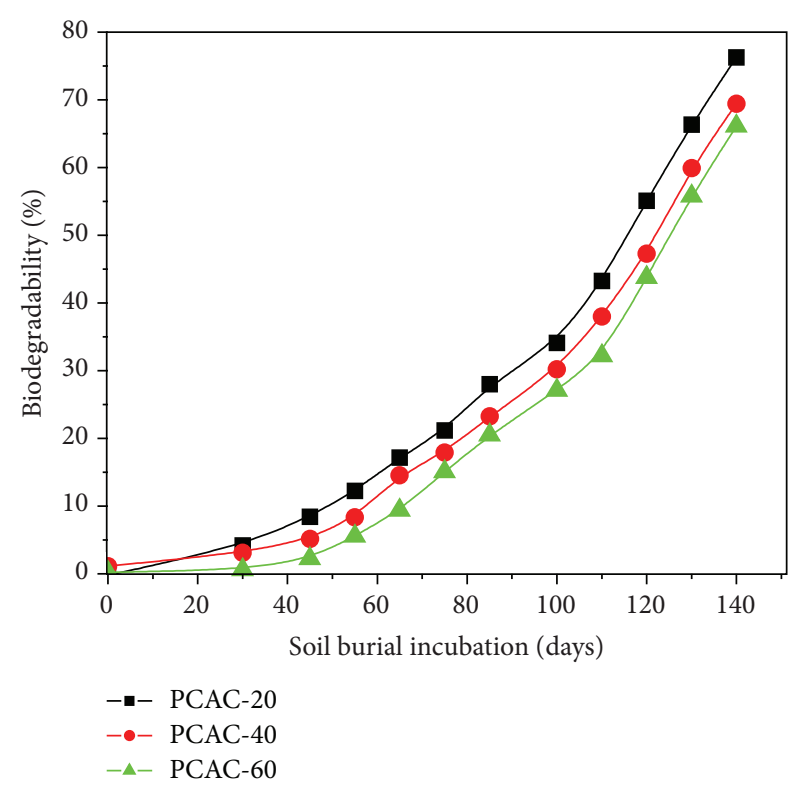

FIGURE 9: Biodegradability of PCACs with soil burial time.

\subsection{Biodegradability}

3.1.1. Film Preparation. To study the biodegradation, PCACs films were prepared by hydraulic press and solution cast method.

3.1.2. Hydraulic Press. PCACs films (thickness of $0.2 \mathrm{~mm}$ ) were prepared in a hydraulic press [15] by pressing the synthesized PCACs between two Teflon plates for a minute under a pressure of $1.5 \mathrm{ton} / \mathrm{cm}^{2}$ below samples melting temperature. The pressed films were stored at the $30^{\circ} \mathrm{C}$ for one week in order to reach the equilibrium crystallinity.

3.1.3. Solution Cast. The PCACs films (thickness of $0.5 \mathrm{~mm}$ ) were prepared by solution casting method $[16,17]$. PCACs sample $(1.0 \mathrm{~g})$ was dissolved in $10 \mathrm{~mL}$ of a chloroform/methanol (9/1) mixture. The solution was cast onto a Petri dish $(d=8.5 \mathrm{~cm})$, and the solvent was allowed to evaporate under atmospheric condition for overnight. The obtained film was dried under vacuum at the $30^{\circ} \mathrm{C}$ for 2 days in order to stabilize crystallinity, before biodegradation experiments.

The weighed solution cast PCACs films were placed in a Petri dish containing $10 \mathrm{~mL}$ PBS ( $\mathrm{pH} 7.2$ and 11.5) and a thermostatically controlled incubator shaker maintained at $37^{\circ} \mathrm{C}$; the films were removed from the PBS at the regular time interval. The films were washed with deionized water and dried under vacuum at the $30^{\circ} \mathrm{C}$ to constant weight. PBS of $\mathrm{pH} 11.5$ contained $7.10 \mathrm{~g}$ of $\mathrm{Na}_{2} \mathrm{HPO}_{4}$ and $0.09 \mathrm{~g}$ of $\mathrm{NaOH}$ in $1 \mathrm{~L}$ of double distilled water $[18,19]$. Degradation at $\mathrm{pH} 7.2$ was carried out at the time interval of 5 days for 50 days, while degradation at $\mathrm{pH} 11.5$ was performed for 28 days.
The extent of hydrolytic degradation was estimated as the (\%) weight loss by

$$
\text { \%Weight loss }=\left\{\left(W_{\mathrm{o}}-W_{t}\right) / W_{0}\right\} \times 100
$$

where $W_{\mathrm{o}}$ is the the weight of original PCACs films and $W_{t}$ is the the weight of residual films after degradation for different time intervals.

Hydraulic pressed PCACs films $(10 \mathrm{~mm} \times 10 \mathrm{~mm} \times$ $0.2 \mathrm{~mm}$ thickness) were placed in Petri dishes containing $10 \mathrm{~mL}$ PBS ( $\mathrm{pH}$ 7.2) with $1 \mathrm{mg}$ Rhizopus delemar lipase. The Petri dishes were then incubated in duplicate at $37^{\circ} \mathrm{C}$, for $96 \mathrm{~h}$, while the media were replaced after $72 \mathrm{~h}$. Blank tests without enzyme were also performed in PBS ( $\mathrm{pH} 7.2$ ) solutions containing PCACs films. After a specific interval of incubation, the films were taken out from the Petri dish, washed with deionized water, and dried under vacuum at $30^{\circ} \mathrm{C}$ to constant weight. Actual degradation by lipase was calculated by subtracting the weight loss, by blank test, from the total weight loss.

The soil burial degradation test [17] of PCACs films was conducted. Hydraulic pressed PCACs films $(15 \mathrm{~mm} \times$ $15 \mathrm{~mm} \times 0.2 \mathrm{~mm}$ thickness) were buried in the soil $(\mathrm{pH} 7.5$, water content capacity $45 \%$ ) in which the relative humidity maintained was 50-60\% (maximum water holding capacity) by spraying water and temperature was maintained at $30^{\circ} \mathrm{C}$ in a humidity chamber (Sonar Co.). The soil used in this study had been taken from the garden of VNIT, Deemed University, Nagpur, India. The soil was conditioned for four weeks before it was used for the actual test; the microbial activity of the soil was tested by using a cotton strip which loses its tensile strength within 10 days of exposure to soil. The buried PCACs films were removed after 28 days at regular interval of seven days. Recovered film was washed with deionized water and dried in vacuum at the $30^{\circ} \mathrm{C}$ to constant weight.

The surface morphology of the original and the degraded PCACs film was examined by scanning electron microscope (JEOL: JXA-840A) equipped with an electron probe microanalyzer system. The films were covered with a golden coating in order to have good conductivity of the electron beam.

\section{Results and Discussion}

PCACs were synthesized by the transesterification polycondensation process from AA, DEC, 1,4-CHDM, and $\mathrm{Ti}(\mathrm{OBu})_{4}$ as transesterification catalyst in the bulk. In PCACs synthesis, the composition of monomer (AA and DEC) was varied in order to obtain PCACs with different composition. The first step of the reaction was carried out at the $160^{\circ} \mathrm{C}$ for $4 \mathrm{~h}$ in the presence of a catalyst $\mathrm{Ti}(\mathrm{OBu})_{4}$ to remove the water and an azeotrope mixture of water-ethanol. In the second step, the reaction was carried out by heating the reaction mixture at the $210^{\circ} \mathrm{C}$ for $1 \mathrm{~h}$ under reduced pressure ( $<0.5$ torr) to remove the water as polycondensation by-product and to make the end product free from water. The various steps involved in the synthesis of the cycloaliphatic PCACs are shown in Scheme 1. All the synthesized cycloaliphatic PCACs were purified by dissolution in chloroform and reprecipitation in 


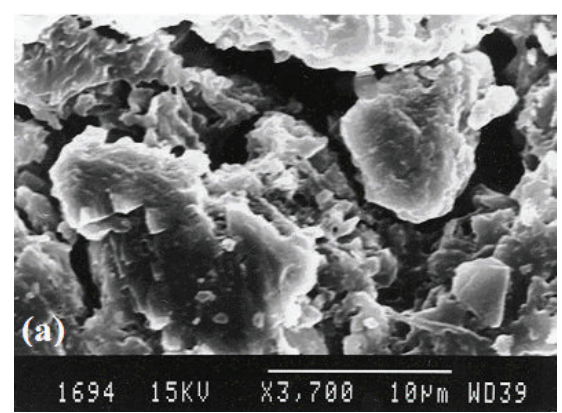

(a)

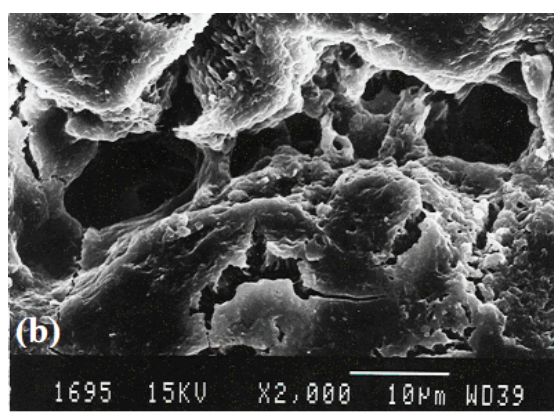

(b)

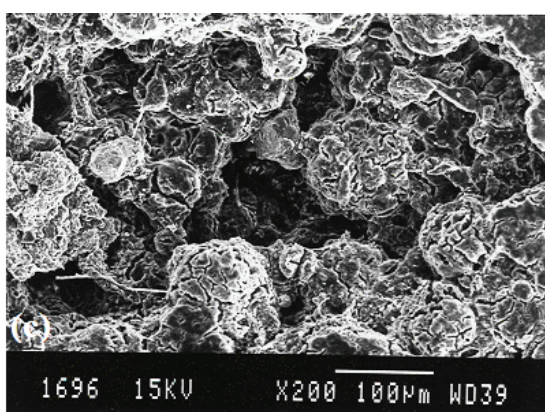

(c)

FIGURE 10: SEM micrographs of soil burial degraded PCAC-60 film after (a) 70, (b) 90, and (c) 144 days.

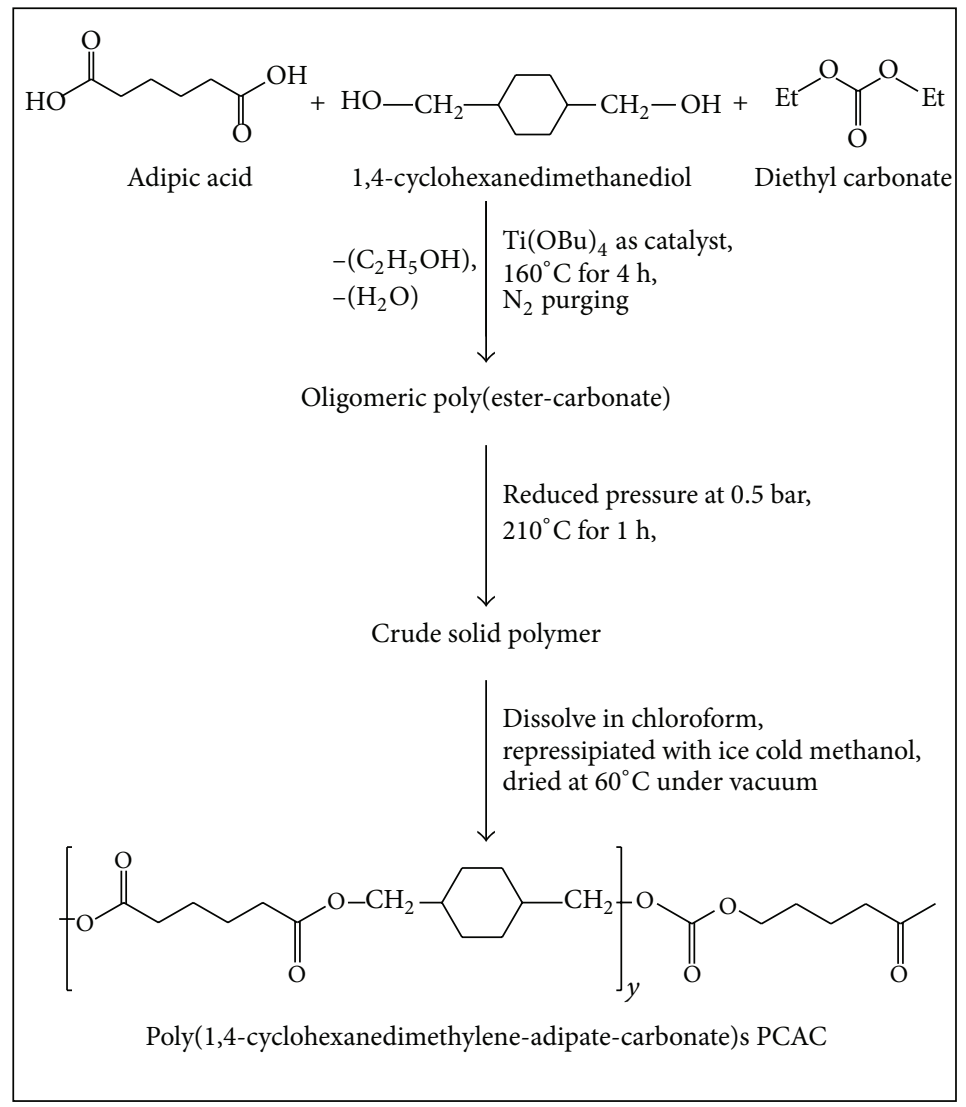

SCHeme 1: Synthetic route and structure synthesized of PCACs.

ice-cold methanol in order to remove the low molecular weight components and excess traces of catalyst.

4.1. Solubility. The W/V solubility behavior of various PCACs was performed and the solubility results are tabulated in Table 1. The solvents used are quite different in nature, which include species that are acidic in nature such as DMSO with strong hydrogen bonding group or strong basic group such as NMP. All the PCACs samples exhibit a good solubility in acetone, THF, DMF, DMSO, NMP, and so forth, which is an important from the application point of view, since it avoids the use of chlorinated solvent in processing such as casting process or microsphere preparations. The PCACs are insoluble in water, methanol, ethanol, ethyl ether, and nhexane, respectively.

4.2. FTIR Spectra. The FTIR spectra of PCACs are shown in Figure 1. The common characteristic strong bands observed in the region at $1726-1735 \mathrm{~cm}^{-1}$ correspond to carbonyl ($\mathrm{C}=\mathrm{O})$ (stretch), which indicates the presence of ester linkage. $\mathrm{O}-\mathrm{H}$ (stretch) at $3433-3442 \mathrm{~cm}^{-1},-\mathrm{CH}_{2}-\mathrm{C}(\mathrm{C}-\mathrm{H}$ stretch $)$ at $2854-2969 \mathrm{~cm}^{-1},-\mathrm{CH}_{2}-\mathrm{C}(\mathrm{C}-\mathrm{H}$ bend $)$ at $1454-1474 \mathrm{~cm}^{-1}$, $\mathrm{C}-\mathrm{C}=\mathrm{O}-\mathrm{O}\left(\right.$ stretch) at $1260-1267 \mathrm{~cm}^{-1}, \mathrm{C}-\mathrm{C}-\mathrm{O}$ (stretch) 
TABLE $1: \mathrm{W} / \mathrm{V} \%$ solubility of PCACs in different solvents at room temperature $\left(30^{\circ} \mathrm{C}\right)$.

\begin{tabular}{lccc}
\hline Solvent & PCAC-20 & PCAC-40 & PCAC-60 \\
\hline Water & - & - & - \\
Chloroform & 98.5 & 99.9 & 100 \\
Acetone & 97.3 & 99.8 & 100 \\
Methanol & - & - & - \\
DMF & 97.9 & 97.3 & 98.5 \\
DMSO & 92.8 & 97.4 & 95.8 \\
THF & 98.0 & 98.1 & 99.1 \\
Hexane & - & - & - \\
Ethyl acetate & 90.7 & 92.3 & 91.8 \\
Ethyl ether & - & - & - \\
Ethanol & - & - & - \\
NMP & 93.6 & 95.4 & 90.2 \\
\hline
\end{tabular}

TABLE 2: Molecular properties of PCACs.

\begin{tabular}{lcccc}
\hline Polymers & $\begin{array}{c}{\left[\eta_{\text {int }}\right]} \\
(\mathrm{dL} / \mathrm{g})\end{array}$ & $\begin{array}{c}M_{n} \\
(\mathrm{~g} / \mathrm{mole})\end{array}$ & $\begin{array}{c}M_{w} \\
(\mathrm{~g} / \mathrm{mole})\end{array}$ & PDI \\
\hline PCAC-20 & 0.39 & 4360 & 4510 & 1.03 \\
PCAC-40 & 0.39 & 4090 & 4210 & 1.02 \\
PCAC-60 & 0.38 & 3840 & 4080 & 1.06 \\
\hline
\end{tabular}

$\left[\eta_{\text {int }}\right]$ : in chloroform using the Tuan-Fouss viscometer at $30^{\circ} \mathrm{C}$.

at $1035-1056 \mathrm{~cm}^{-1}$, and C-O-C (stretch) at $1163-1178 \mathrm{~cm}^{-1}$, respectively [12].

4.3. Molecular Weight and Intrinsic Viscosity. The molecular weight data and intrinsic viscosity of the synthesized PCACs are presented in Table 2. The number average molecular weights $\left(M_{n}\right)$ of PCACs are in the range of 3840 to $4360 \mathrm{~g} / \mathrm{moL}$, while the polydispersity index $\left(M_{w} / M_{n}\right)$ is nearly uniform showing active polymer. The intrinsic viscosity $\left[\eta_{\text {int }}\right]$ of the PCACs was determined by the Huggins equation for dilute solution. It is found that the intrinsic viscosities of PCACs lie between 0.38 and $0.39 \mathrm{dL} / \mathrm{g}$. The intrinsic viscosity is lower for PCAC-60 in comparison with PCAC-20 and PCAC-40 because of the existence of branching in the polymer chain.

4.4. Thermal Studies. The DSC thermograms of PCACs with melting endotherms are presented in Figure 2. The endothermic peaks observed are attributed to the melting of PCACs crystals. The PCACs have a melting point in the range of $85.9-100.6^{\circ} \mathrm{C}$. However, the melting point of PCAC-20 and PCAC-60 shifted towards the higher temperature compared to PCAC-40.

Figure 3 shows the TG thermogram of the PCACs. The thermal decomposition of PCAC starts after $250^{\circ} \mathrm{C}$ and the fractional weight loss of the PCAC was less than $50 \%$ at $350^{\circ} \mathrm{C}$. In order to compare the relative thermal stability of polymer, the temperatures for weight losses of $10 \%, 50 \%$ and $90 \%$ are presented in Table $3.10 \%$ of the weight losses are considered to represent the beginning of mass loss. It can be observed that $10 \%$ of the weight losses are above $270^{\circ} \mathrm{C}$ in all polymers. From the weight loss data, it is observed that the thermal stability of PCACs was good. Also from thermal properties, it was observed that no appreciable thermal degradation takes place during synthesis, so there is no need to use a heat stabilizer during the synthesis PCACs.

4.5. X-Ray Analysis. X-ray diffraction pattern for the PCACs is shown in Figure 4. The characteristic peaks for PCACs peaks were seen at a $2 \theta$ value of $19-30^{\circ}$ for all the poly(ester carbonate)s. It can be seen that in PCAC- 40 and PCAC60 the peak positions are at higher $2 \theta$ values than PCAC20 which can be assigned to a small decrease of the unit cell dimension. The peak positions in the diffractograms of the PCACs show that there is no change in the type of the crystal lattice. The degrees of crystallinity were $46.67 \%$, $71.75 \%$, and $54.72 \%$ for PCAC-20, PCAC-40, and PCAC60 , respectively, calculated from XRD measurements. The lower crystallinity observed for PCAC-20 compared with PCAC-40 and PCAC-60 is due to the consequence of possible distortions in the crystal lattice and content of cyclic diol. Usually lower crystallinity favors biodegradation.

\subsection{Biodegradation Studies}

4.6.1. Hydrolytic Degradation. The hydrolytic degradation behavior of PCACs in PBS of pH 7.2 and 11.5 at $37^{\circ} \mathrm{C}$ is shown in Figures 5(a) and 5(b). A linear increase in hydrolytic weight loss was observed with incubation time at $\mathrm{pH}$ 7.2. However, at $\mathrm{pH} 11.5$ weight loss was initially more, while afterwards rate of weight loss decreases. It was observed that the film becomes brittle on incubation and the degradation was faster under alkaline condition at $\mathrm{pH}$ 11.5. The hydrolytic weight loss of the PCAC-60 and PCAC-40 was lower than the weight loss of PCAC-20. After 28 days of hydrolytic degradation, PCAC20, PCAC-40, and PCAC-60 showed a 70\%, 65\%, and $60 \%$ mass loss at $\mathrm{pH} 11.5$, respectively, whereas after 50 days of hydrolytic degradation in PBS at pH 7.2, PCAC-20, PCAC40, and PCAC-60 showed a 34\%, 32\%, and 31.4\% weight loss, respectively.

The $\left[\eta_{\text {int }}\right]$ of hydrolytic degraded PCACs film was performed and the results are shown in Figure 5(c). The $\left[\eta_{\text {int }}\right]$ of the PCACs decreased slightly after hydrolytic degradation with incubation time. The $\left[\eta_{\text {int }}\right]$ decrease followed a linear profile whereby the rate of decrease of $\left[\eta_{\text {int }}\right]$ was observed to be more in PCAC-20 as compared to PCAC-40 and PCAC60 , respectively. The similar results were observed on average molecular weight estimation of degraded samples by end group analysis. From Figure 5(d), it is observed that, with the increase of incubation time (days), the $M_{n}$ values were found to be reduced. The variation of $\left[\eta_{\text {int }}\right]$ and molecular weight indicated that the increase in degradation with the incubation time may give rise to the formation of low molecular weight molecules and oligomers.

The SEM micrographs of the surface of the PCACs films after hydrolytic degradation are shown in Figure 6. The morphology of the PCACs film changes upon degradation. After 40 days of hydrolytic degradation, the surfaces of films 
TABLE 3: Thermal properties of PCACs.

\begin{tabular}{lcccccc}
\hline \multirow{2}{*}{ Polymers } & $T_{g}$ & $T_{m}$ & $T_{d}$ & \multicolumn{2}{c}{$\Delta H_{m}$} & \multicolumn{2}{c}{ Temperatures $\left({ }^{\circ} \mathrm{C}\right)$ of weight loss for } \\
& $\left({ }^{\circ} \mathrm{C}\right)$ & $\left({ }^{\circ} \mathrm{C}\right)$ & $\left({ }^{\circ} \mathrm{C}\right)$ & $(\mathrm{J} / \mathrm{g})$ & $10 \%$ & $50 \%$ \\
\hline PCAC-20 & -24.8 & 100.6 & 400 & 57.7 & 270 & $30 \%$ \\
PCAC-40 & -29.5 & 85.9 & 400 & 34.2 & 275 & 375 \\
PCAC-60 & -28.8 & 101.9 & 450 & 16.9 & 280 & 375 \\
\hline
\end{tabular}

TABLE 4: Thermal properties of PCACs after enzymatic hydrolysis.

\begin{tabular}{|c|c|c|c|c|c|}
\hline Polymer & Incubation time $(\mathrm{h})$ & $T_{g}\left({ }^{\circ} \mathrm{C}\right)$ & $T_{m}\left({ }^{\circ} \mathrm{C}\right)$ & $\Delta H_{m}(\mathrm{~J} / \mathrm{g})$ & $X_{c}(\%)$ \\
\hline \multirow{4}{*}{ PCAC-20 } & 0 & -24.8 & 100.6 & 57.7 & 46.67 \\
\hline & 24 & -24.6 & 100.6 & 57.8 & - \\
\hline & 72 & -24.2 & 101.4 & 58.2 & 49.53 \\
\hline & 144 & -24.4 & 101.7 & 58.6 & 51.75 \\
\hline \multirow{4}{*}{ PCAC- 40} & 0 & -17.5 & 85.9 & 34.2 & 51.75 \\
\hline & 24 & -17.9 & 86.0 & 34.2 & - \\
\hline & 72 & -17.2 & 86.2 & 34.5 & 53.54 \\
\hline & 144 & -17.6 & 86.8 & 34.8 & 56.55 \\
\hline \multirow{4}{*}{ PCAC-60 } & 0 & -8.8 & 101.9 & 16.9 & 54.72 \\
\hline & 24 & -8.8 & 102.1 & 16.9 & - \\
\hline & 72 & -9.1 & 102.2 & 17.1 & 55.74 \\
\hline & 144 & -9.2 & 102.2 & 17.9 & 56.62 \\
\hline
\end{tabular}

show surface irregularities and small holes in a buffer solution and it increases with an increase in the exposure time.

Figure 7(a) shows the normalized weight loss per unit area of the PCACs film as a function of time during enzymatic degradation. A slight variation in weight loss is observed during the first few hours of biodegradation (16-48 hours) at a slower rate, while afterwards abrupt change in weight loss continues. This might be enzymes attack on the surface of an insoluble substrate via surface erosion. Thus, hydrolysis rates are decreased after consumption of the amorphous material of the surface. Afterwards, a layer of crystalline domains remains, where only a slow degradation may occur. This inhibits penetration of water into the body of the film resulting in decreased rates of hydrolysis. In addition of fresh lipase after $72 \mathrm{~h}$ during incubation of film, the rate of enzymatic hydrolysis increases by more attacks of enzyme on the surface of the polymer film. The enzymatic degradation of PCACs follows as the order: PCAC-20 > PCAC-40 > PCAC60, respectively. PCAC-20 and PCAC-40 exhibit higher weight loss value, compared to PCAC-60. This may be due to low \% carbonate content of PCACs.

From the Figure 7(b), the average molecular weight of degraded PCACs slightly decreases with the increase in incubation time. The reduction of $M_{n}$ values may be due to a scission of ester bonds in the PCACs. Also, similar changes in $\left[\eta_{\text {int }}\right]$ of degraded PCACs were observed in Figure 7(c). DSC and XRD data of enzymatically degraded PCACs samples are presented in Table 4. Results show that, as the time of hydrolysis increases, $T_{m}$ of degraded sample very slightly increases; similar type of changes was observed in $\Delta H_{m}$ data. XRD results show the small increase in the degree of crystallinity of degraded samples, which may be due to the loss of an amorphous mass of the sample during degradation and afterwards only crystalline mass was left and hence crystallinity increases with degradation.

SEM micrographs of PCAC-60 after (a) $96 \mathrm{~h}$ and (b) $144 \mathrm{~h}$ of enzymatic degradation are presented in Figure 8. One can observe that the surface of the original film changes upon degradation. The original film was smooth and homogeneous. After 96 hours, degradation occurs on the film surface, making the surface rough by forming flaky structure, holes, and irregularity on it and after increasing incubation time, surface irregularity becomes deeper and deeper. Especially after increasing the exposure time, there were indications that the fragments had been removed from the surface. It is clear that the action of Rhizopus delemar lipase led to the formation of a rough surface with large cracks, holes, which also extend to a large depth within the film mass with an increase in incubation time. It is clear that biodegradation depends strongly on the molecular weight, polymer structure, and crystallinity of the polymers, as the effect of the enzymes is more pronounced in PCAC-20 and PCAC-40 compared to PCAC-60, verifying that degradation occurs preferably at amorphous regions.

4.6.2. Soil Burial Degradation. Figure 9 shows the biodegradability of PCACs in soil burial degradation at $30^{\circ} \mathrm{C}$. Both the soil burial degradation and hydrolytic degradation process show similar trends for degradation of PCACs. There is a difference in their degradation mechanism, as the effect of microorganisms arises on the surface of the polymer film in the case of soil burial. It can be seen that the biodegradability of PCACs initially commences with surface erosion, followed by random chain scission of the polymer main chain from 
TABLE 5: Molecular weights, thermal properties, and crystallinity change after soil burial degradation of PCACs.

\begin{tabular}{|c|c|c|c|c|c|}
\hline Polymer & Incubation time (days) & $M_{n}(\mathrm{~g} / \mathrm{mole})$ & $T_{m}\left({ }^{\circ} \mathrm{C}\right)$ & $\Delta H_{m}(\mathrm{~J} / \mathrm{g})$ & $X_{c}(\%)$ \\
\hline \multirow{3}{*}{ PCAC-20 } & 28 & 4360 & 100.6 & 57.7 & 47.4 \\
\hline & 70 & 1800 & 103 & 64.3 & 57.9 \\
\hline & 144 & 750 & 106 & 73.1 & 65.2 \\
\hline \multirow{3}{*}{ PCAC- 40} & 28 & 4090 & 85.9 & 34.2 & 54 \\
\hline & 70 & 2000 & 90 & 40.9 & 58.2 \\
\hline & 144 & 880 & 93 & 47.1 & 65.6 \\
\hline \multirow{3}{*}{ PCAC-60 } & 28 & 3840 & 101.9 & 16.9 & 55.4 \\
\hline & 70 & 1600 & 105 & 18.5 & 60.5 \\
\hline & 144 & 900 & 107 & 19.8 & 66.9 \\
\hline
\end{tabular}

the attached soil microbes [20,21]. The decreased molecular weight of PCACs may be attributed to the hydrolysis of the aliphatic ester linkage of PCACs, conversion to oligomer and monomer. The soil conditions are suitable for microorganism growth due to the high humidity, temperature of $30^{\circ} \mathrm{C}$, and $\mathrm{pH}$ of 7.5. The DSC and XRD data of soil degraded sample shows that the $T_{m}, \Delta H_{m}$, and crystallinity of the samples increase with incubation time as tabulated in Table 5. The weight loss of PCACs decreased more rapidly in the soil burial test. This indicates that the microorganisms present in natural compost soil are more numerous because of the additional nourishment content in a soil environment. It may also be seen that the metabolism rate of microorganisms in natural soil is much higher [21, 22].

The SEM micrographs of soil burial degraded PCAC-60 film after 70, 90, and 144 days are presented in Figure 10. From SEM, one can observe that the morphology shows a large number of holes, cracks, cavities, and surface irregularities. The numbers of cracks and small holes were becoming deeper with increasing the exposure time and fragments had been removed from surface to increase in length of cracks and appearance of cavities. In case of soil burial degraded samples, the formation of holes and cracks enhancing fragmentation in polymeric surface may due to more attacks of microorganism.

\section{Conclusions}

Biodegradable aliphatic/alicyclic PECs were successfully synthesized via transesterification polycondensation process. The structure of synthesized PCACs was confirmed by FTIR studies. The degradation of the PCACs shows that the PCAC-20 and PCAC-40 exhibit higher weight loss, whereas PCAC-60 exhibits lower weight loss due to low melting point, crystallinity, and the carbonate content (\%). Enzymatic degradation using Rhizopus delemar lipase and soil burial degradation showed that the biodegradability was enhanced with the decrease in the crystallinity of PCACs, and also the films become brittle on incubation. From the studies, it was concluded that molecular weight has much less effect on degradation rates than a physical structure. SEM microphotographs of the soil burial test demonstrated that degradation occurred all over the surface of PCACs films creating holes and cracks. This was attributed to the diffusion rates in the main body of the polymer films and also into the crystalline domains of the PCACs. Biodegradation rate of PCACs follows the following order: PCAC-20 > PCAC-40 $>$ PCAC-60.

\section{Conflict of Interests}

The authors declare that there is no conflict of interests regarding the publication of this paper.

\section{Acknowledgment}

The authors are thankful to the Ministry of Human Resources Development, New Delhi, India, for their financial assistance through Project no. F: 27-16/2003-TS.

\section{References}

[1] H. Tian, Z. Tang, X. Zhuang, X. Chen, and X. Jing, "Biodegradable synthetic polymers: preparation, functionalization and biomedical application," Progress in Polymer Science, vol. 37, no. 2, pp. 237-280, 2012.

[2] G. Scott, “'Green' polymers," Polymer Degradation and Stability, vol. 68 , no. 1, pp. 1-7, 2000.

[3] L. S. Nair and C. T. Laurencin, "Biodegradable polymers as biomaterials," Progress in Polymer Science, vol. 32, no. 8-9, pp. 762-798, 2007.

[4] Y. Tsai, L. C. Jheng, and C. Y. Hung, "Synthesis, properties and enzymatic hydrolysis of biodegradable alicyclic/aliphatic copolyesters based on 1,3/1,4-cyclohexanedimethanol," Polymer Degradation and Stability, vol. 95, no. 1, pp. 72-78, 2010.

[5] C. F. Wang, Y. X. Lin, T. Jiang, F. He, and R. X. Zhuo, "Polyethylenimine-grafted polycarbonates as biodegradable polycations for gene delivery," Biomaterials, vol. 30, no. 27, pp. 4824-4832, 2009.

[6] J. Yang, Q. Hao, X. Liu, C. Ba, and A. Cao, "Novel biodegradable aliphatic poly(butylene succinate-co-cyclic carbonates)s with functionalizable carbonate building blocks. 1. Chemical synthesis and their structural and physical characterization," Biomacromolecules, vol. 5, no. 1, pp. 209-218, 2004.

[7] X. Yu, R. Zhuo, J. Feng, and J. Liao, "Enzymatic ring-opening polymerization of 2,2-dimethyltrimethylene carbonate catalyzed by PPL immobilized on silica nanoparticles," European Polymer Journal, vol. 40, no. 11, pp. 2445-2450, 2004.

[8] E. Ranucci, Y. Liu, M. S. Lindblad, and A.-C. Albertsson, "New biodegradable polymers from renewable sources. High 
molecular weight poly(ester carbonate)s from succinic acid and 1,3-propanediol," Macromolecular Rapid Communications, vol. 21, no. 10, pp. 680-684, 2000.

[9] Y. Liu, E. Ranucci, M. S. Lindblad, and A. C. Albertsson, "New biodegradable polymers from renewable sources: Polyester-carbonates based on 1,3-propylene-co-1,4cyclohexanedimethylene succinate," Journal of Polymer Science A: Polymer Chemistry, vol. 39, no. 14, pp. 2508-2519, 2001.

[10] S. Matsumura, K. Tsukada, and K. Toshima, "Novel lipasecatalyzed ring-opening copolymerization of lactide and trimethylene carbonate forming poly(ester carbonate)s," International Journal of Biological Macromolecules, vol. 25, no. 1-3, pp. 161-167, 1999.

[11] M. Yokoe, A. O. I. Keigo, and M. Okada, "Biodegradable polymers based on renewable resources. VII. Novel random and alternating copolycarbonates from 1,4:3,6-dianhydrohexitols and aliphatic diols," Journal of Polymer Science A: Polymer Chemistry, vol. 41, no. 15, pp. 2312-2321, 2003.

[12] S. S. Umare, A. S. Chandure, and R. A. Pandey, "Synthesis, characterization and biodegradable studies of 1,3-propanediol based polyesters," Polymer Degradation and Stability, vol. 92, no. 3, pp. 464-479, 2007.

[13] E. Su, M. Zhang, J. Zhang, J. Gao, and D. Wei, "Lipase-catalyzed irreversible transesterification of vegetable oils for fatty acid methyl esters production with dimethyl carbonate as the acyl acceptor," Biochemical Engineering Journal, vol. 36, no. 2, pp. 167-173, 2007.

[14] G. Z. Papageorgiou, S. G. Nanaki, and D. N. Bikiaris, "Synthesis and characterization of novel poly(propylene terephthalate-coadipate) biodegradable random copolyesters," Polymer Degradation and Stability, vol. 95, no. 4, pp. 627-637, 2010.

[15] D. N. Bikiaris and G. P. Karayannidis, "Synthesis and characterisation of branched and partially crosslinked poly(ethylene terephthalate)," Polymer International, vol. 52, no. 7, pp. 12301239, 2003.

[16] F. He, S. Li, M. Vert, and R. Zhuo, "Enzyme-catalyzed polymerization and degradation of copolymers prepared from $\varepsilon$ caprolactone and poly(ethylene glycol)," Polymer, vol. 44, no. 18, pp. 5145-5151, 2003.

[17] H. Shinoda, Y. Asou, T. Kashima, T. Kato, Y. Tseng, and T. Yagi, "Amphiphilic biodegradable copolymer, poly(aspartic acid-colactide): acceleration of degradation rate and improvement of thermal stability for poly(lactic acid), poly(butylene succinate) and poly(-caprolactone)," Polymer Degradation and Stability, vol. 80, no. 2, pp. 241-250, 2003.

[18] X. J. Loh, K. Tan, X. Li, and J. Li, "The in vitro hydrolysis of poly(ester urethane)s consisting of poly[(R)-3hydroxybutyrate] and poly(ethylene glycol)," Biomaterials, vol. 27, no. 9, pp. 1841-1850, 2006.

[19] F. Kondratowicz and R. Ukielski, "Synthesis and hydrolytic degradation of poly(ethylene succinate) and poly(ethylene terephthalate) copolymers," Polymer Degradation and Stability, vol. 94, no. 3, pp. 375-382, 2009.

[20] H. Tsuji and K. Suzuyoshi, "Environmental degradation of biodegradable polyesters 1 . Poly( $\varepsilon$-caprolactone), poly[(R)-3hydroxybutyrate], and poly(L-lactide) films in controlled static seawater," Polymer Degradation and Stability, vol. 75, no. 2, pp. 347-355, 2002.
[21] J. Ratto, J. Stenhouse, M. Auerbach, J. Mitchell, and R. Farrell, "Processing, performance and biodegradability of a thermoplastic aliphatic polyester/starch system," Polymer, vol. 40, no. 24, pp. 6777-6788, 1999.

[22] H. S. Kim, H. J. Kim, J. W. Lee, and I. G. Choi, "Biodegradability of bio-flour filled biodegradable poly(butylene succinate) biocomposites in natural and compost soil," Polymer Degradation and Stability, vol. 91, no. 5, pp. 1117-1127, 2006. 

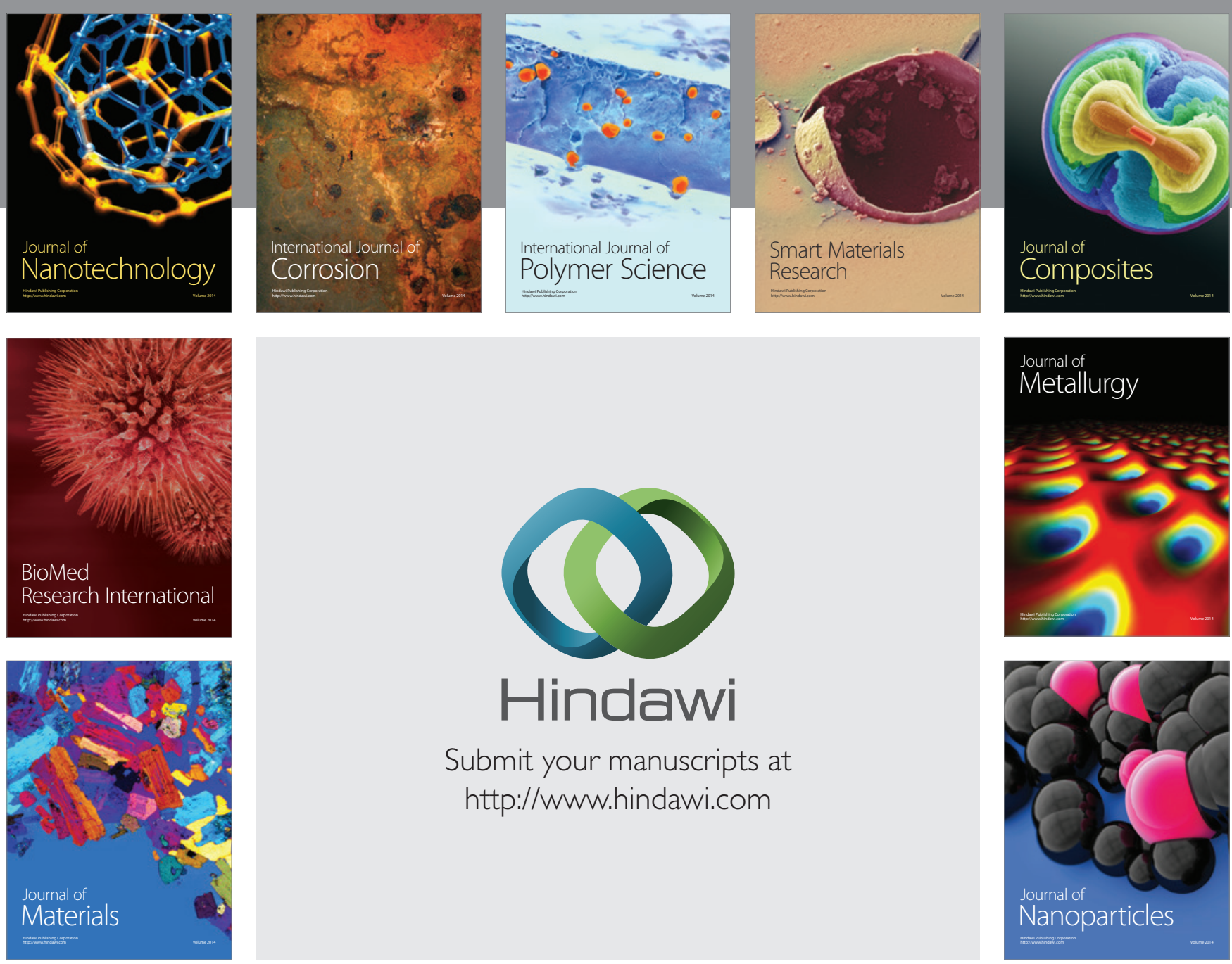

Submit your manuscripts at http://www.hindawi.com
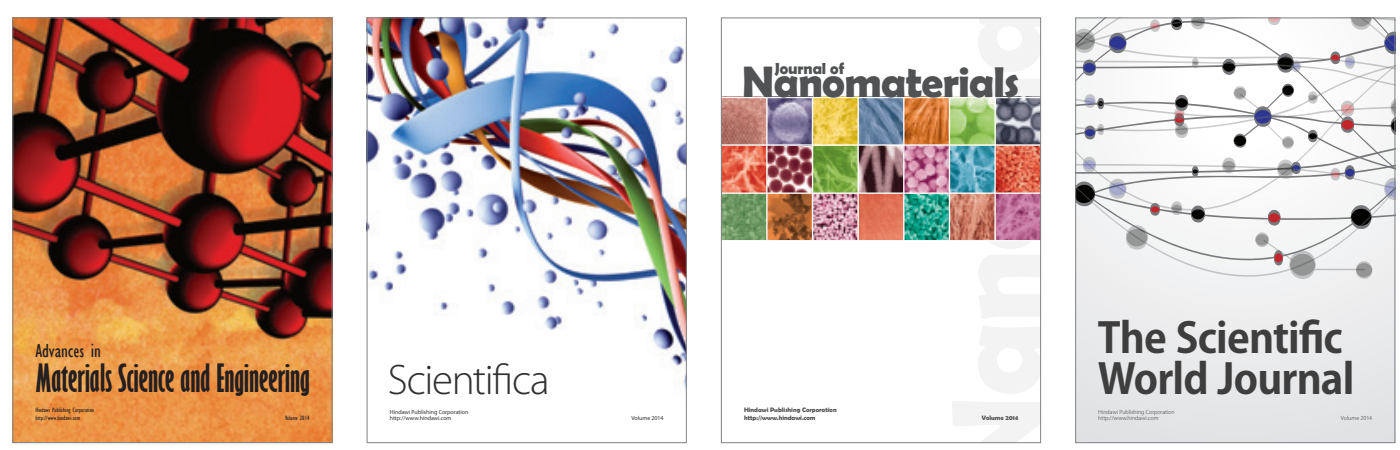

\section{The Scientific World Journal}
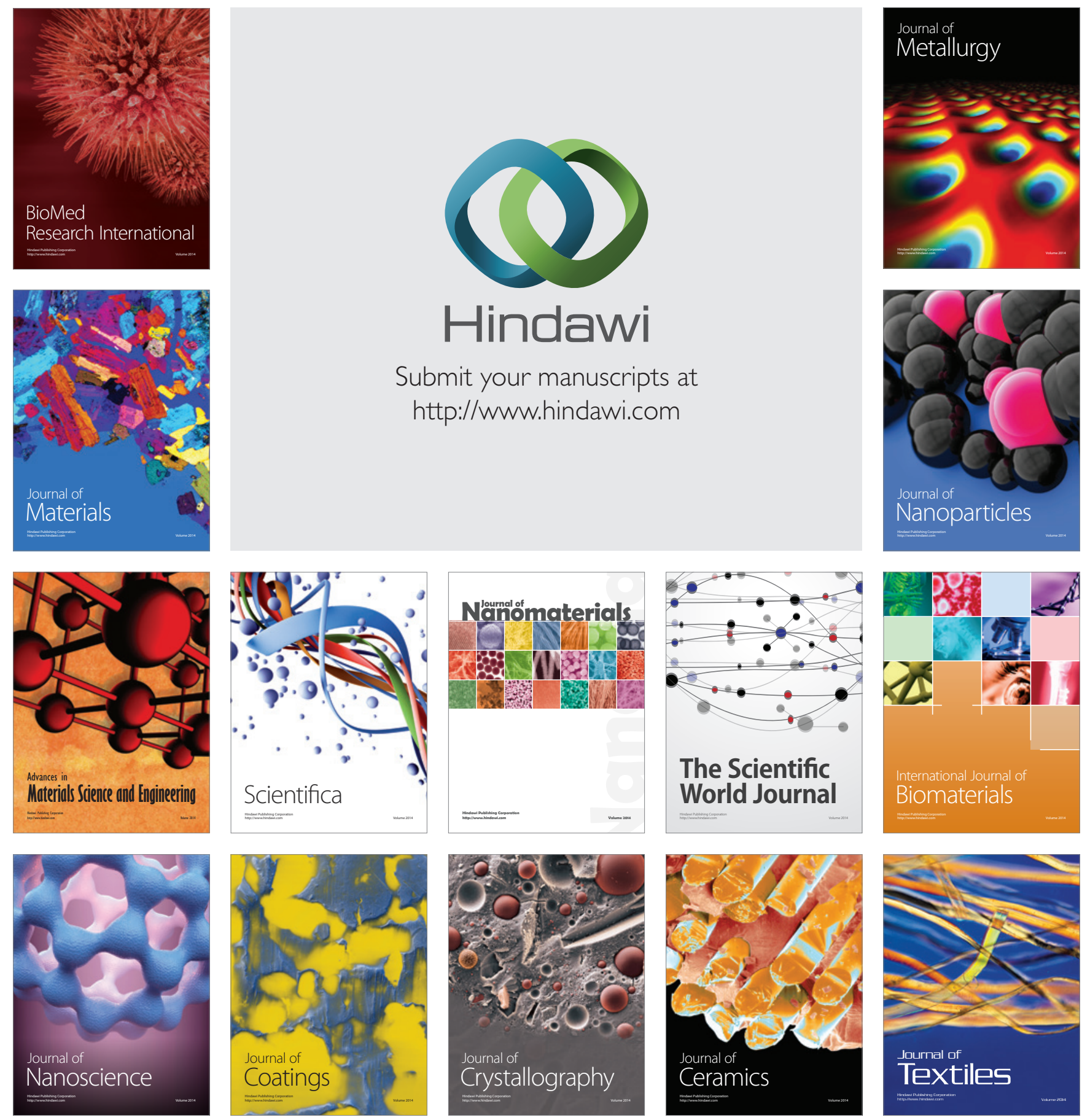